\title{
REMARK ON A THEOREM OF D. H. FREMLIN CONCERNING K-ANALYTIC HAUSDORFF SPACES
}

\author{
by KEITH J. DEVLIN' \\ (Received 2nd May 1977)
}

\section{Introduction}

At the 29th Meeting of the British Mathematical Colloquium held at Edinburgh in March 1977, D. H. Fremlin announced the following result.

Let $\Phi$ denote the proposition that there exists a $K$-analytic Hausdorff space, having metrizable compacta, which is not Souslin. (We refer the reader to Fremlin's paper (1) for the relevant definitions)

Theorem 1.1. (Fremlin) (i) Assume $2^{\aleph_{0}}=\aleph_{1}$. Then $\Phi$ is valid.

(ii) Assume Martin's Axiom together with $2^{\aleph_{0}}>\aleph_{1}$. Then $\Phi$ is false.

Corollary 1.2. (Fremlin) $\Phi$ cannot be decided on the basis of the usual (ZermeloFraenkel) axioms of set theory.

Now, it is well known that whereas the continuum hypothesis $-2^{\kappa_{0}}=\kappa_{1}$-is a very powerful assumption, its negation is rather a weak statement. Martin's Axiom, on the other hand, is a consequence of the continuum hypothesis, but in the presence of the negation of the continuum hypothesis is an extremely powerful assumption, many of whose consequences are in direct contradiction with consequences of $2^{\aleph_{0}}=\aleph_{1}$. The consistency of the combination of Martin's Axiom and $2^{\kappa_{0}}>\aleph_{1}$ is known. In Fremlin's proof of part (ii) of Theorem 1.1, Martin's Axiom is used essentially. Thus it is of interest to know whether the result needs Martin's axiom, or whether the truth or falsity of $\Phi$ depends simply upon the truth or falsity of the continuum hypothesis. In this case, as in almost all others, one would expect the answer to be "no", but such a question deserves to be settled properly. In this note we do just this: namely we prove the following result:

Theorem 1.3. The falsity of $\Phi$ cannot be established on the basis of the axioms of set theory plus the negation of the continuum hypothesis.

Indeed, even if we assume any "axiom" such as $2^{\aleph_{0}}=\aleph_{2}, 2^{\aleph_{0}}=\aleph_{100}$, etc., we cannot prove that $\Phi$ fails.

\footnotetext{
'The result in this paper was proved during our attendance at the BMC meeting at Edinburgh (March 1977), following a lecture given by Fremlin at the meeting. We wish to thank the organisers of that meeting for their efforts and their hospitality.
} 
After hearing Fremlin's lecture, we asked the question raised above, and, as well as conjecturing the correct answer to our question, Fremlin remarked (with absolute justification) that this question could only be answered by a mathematical logician, and that logicians rarely publish answers to questions of this nature: the result being that the analyst is presented with a bald fact, unaccompanied by any hint of the reasoning behind that fact. Having been ourselves guilty of this in the past, we felt it would be of assistance to devote a little time to writing up the proof. The result is this note. To the expert in the techniques involved in obtaining independence results, what we do does not involve anything greatly different from many other independence proofs, but hopefully the paper will prove illuminating to the analyst not versed in such sorcery.

As fundamental we take the Zermelo-Fraenkel system of set theory. More loosely, we adopt the "usual" set theory underlying mathematics, including the Axiom of Choice. Our notation is that current in set theoretical circles. The ordinals are defined in the von Neumann manner, with every ordinal equal to the set of all its predecessors. We reserve lower case Greek letters to denote arbitary ordinal numbers. The first infinite ordinal (i.e. the set of all natural numbers) is denoted by $\omega$, the first uncountable ordinal by $\omega_{1}$, the first ordinal not equinumerous with any ordinal $\alpha \leqslant \omega_{1}$ by $\omega_{2}$, the first ordinal not equinumerous with any $\alpha \leqslant \omega_{2}$ by $\omega_{3}$, and so on. The ordinals $\omega, \omega_{1}, \omega_{2}, \ldots$ are the infinite cardinals; considered in its role as a cardinal number, we usually write $\aleph_{0}$ in place of $\omega$ and $\aleph_{n}$ in places of $\omega_{n}$. The cardinality of any set $X$ is denoted by $|X|$. Thus a set $X$ will be countable iff $|X| \leqslant \aleph_{0}$, and uncountable iff $|X| \geqslant \aleph_{1}$.

\section{The method of boolean extensions}

In order that our paper may be accessible to a fairly wide range of readers, we present here an extremely brief account of the main method used to obtain independence results. For more details we refer the reader to (2).

Let $V$ denote the collection of all sets (the set-theoretic universe). Now, there is a one-many correspondence between sets and characteristic functions, so if we are prepared to accept the presence of many functions representing the same set (because they have different domains), we can replace $V$ by an "equivalent" collection of characteristic functions. Now suppose $B$ is any complete boolean algebra. Suppose that we define a "universe" $V^{B}$ now consisting not of functions which take only the values 0 and 1 (as do characteristic functions), but of functions whose range is a subset of B. For two "sets" in $V^{\mathrm{B}}$, it will not now always be the case that they are equal or distinct, but there will be some member of $B$ which represents the degree to which they are equal. In a natural way, this induces a boolean truth valuation for any set theoretical assertion $\phi$, denoted by $\|\phi\|$. Thus $\|\phi\|$ represents the amount (in the sense of B) by which $\phi$ is true in the "universe" $V^{\mathrm{B}}$. (As the reader may suspect, this all works out correctly by virtue of the fact that boolean algebras behave in the same way as do "truth tables".) Now, it can be proved that if $\phi$ is a theorem of Zermelo-Fraenkel set theory, then, regardless of the actual choice of $B,\|\phi\|=1$, and if $\phi$ is provably false in Zermelo-Fraenkel set theory, then $\|\phi\|=0$. Hence, if we can find an algebra $B$ for which $0<\|\phi\|<1$, then $\phi$ will be proved to be undecidable! In 
our present case, with $\Phi$ the assertion of Section 1, we know that $\Phi$ follows from $2^{\aleph_{0}}=\aleph_{1}$ (in set theory), and it suffices to find an algebra B for which $\|\phi\|>0$, were $\phi$ is the conjunction of the assertions $\Phi$ and $2^{\aleph_{0}}>\aleph_{1}$, for this will imply that the implication

$$
2^{\aleph_{0}}>\aleph_{1} \Rightarrow \text { not }-\Phi
$$

cannot be proved in set theory, which is what we require. (As might be expected, for any statement $\phi$, $\|$ not $-\phi\|=1-\| \phi \|$. Indeed, boolean values combine in precisely the manner one would expect.) In fact, in order to prove that $\|\phi\|>0$, we shall need to assume the continuum hypothesis. That this will still give the required consistency result follows from the fact that the continuum hypothesis is itself consistent with Zermelo-Fraenkel set theory. Of course, having once decided on the algebra $B$ to be used, we shall be faced with the problem of calculating $\|\phi\|$ for the relevant assertion $\phi$, and this will require some knowledge of how boolean truth values are defined. We indicate briefly the (natural) definition here.

Since elements of $V^{B}$ are B-valued "characteristic functions", if $u \in V^{B}$ and $x \in \operatorname{dom}(u)$, then $u(x)$ represents the truth value of $x$ being a member of $u$. This enables us to define the truth values $\|u \in v\|$ and $\|u=v\|$ for elements $u, v$ of $V^{B}$. These definitions are quite natural, but we do not give them in full here. (In fact the most "obvious" definition, setting $\mid u \in v \|=v(u)$, does not work, since $\|u \in v\|$ needs to be defined for all $u, v$, not just when $u$ is in $\operatorname{dom}(v)$. The reader is urged to refer to (2) if he wishes to understand fully these boolean valued notions.) Having defined truth values for such atomic sentences, we can define $\|\phi\|$ for any $\phi$ by induction on the construction of $\phi$. (Being a set theoretical assertion, $\phi$ will only contain such atomic subformulas, built up using quantifiers and logical connectives). The schemata are as follows:

$$
\begin{aligned}
& \| \text { not }-\phi\|=-\| \phi \| \text {; } \\
& \| \phi \text { or } \psi\|=\| \phi\|\vee\| \psi \| \text {; } \\
& \| \phi \text { and } \psi\|=\| \phi\|\wedge\| \psi \| \text {; } \\
& \|\exists u \phi(u)\|=\bigvee_{u \in V^{\delta}}\|\phi(u)\| ; \\
& \|\forall u \phi(u)\|=\bigwedge_{u \in V^{\theta}}\|\phi(u)\| .
\end{aligned}
$$

Of course, for any algebra $B, V^{B}$ is just a collection of sets (in set theory, everything is a set), and hence $V^{B}$ is a subcollection of $V$. We may, nevertheless, regard $V^{\mathrm{B}}$ as an extension of $V$. Indeed, we may define a subcollection $\check{V}$ of $V^{\mathrm{B}}$, isomorphic to $V$ (in an obvious, characteristic function sense) by defining, for $x \in V$, the element $\check{x}$ of $V^{B}$ having domain $\{\check{y} \mid y \in x\}$, such that $\check{x}(\check{y})=1$ for all $y \in x$. (This is really a definition by induction on the well-founded relation $\in$.) The presence of the copy, $\check{V}$, of $V$ inside $V^{B}$ will be very useful to us in the ensuing development.

\section{The main theorem}

During his BMC talk, Fremlin sketched a proof of the following theorem. 
Theorem 3.1. (Fremlin) Let $X$ denote the space $\omega^{\omega}$ endowed with the product topology induced by the discrete topology on $\omega$. (It is well known that $X$ is homeomorphic to the irrationals.) Then $\Phi$ is equivalent to $\Psi$, where $\Psi$ is the following assertion:

there is an uncountable set $A \subseteq X$ such that $A \cap K$ is countable for every compact set $K \subseteq X$.

Indeed, Fremlin's proof of 1.1 proceeds by establishing the analogous result with $\Psi$ in place of $\Phi$. We shall prove 1.3 by finding a complete boolean algebra B such that $\left\|2^{\aleph_{0}}>\aleph_{1}\right\|=1$ and $\|\Psi\|=1$. By modifying the proof, we could obtain more specific results such as $\left\|2^{\aleph_{0}}=\aleph_{13}\right\|=1$ and $\|\Psi\|=1$, and indeed the expert in such techniques will see this at once, but we do not give any details here.

We commence by describing the algebra B. Let $I=2^{\omega \times \omega_{2}}$ have the product topology determined by the discrete topology on $2(=\{0,1\})$. Let $C$ be the algebra of all Borel subsets of $I$, and let $\Delta$ be the ideal of all meagre Borel sets. $C$ is a $\sigma$-algebra and $\Delta$ a $\sigma$-ideal, but since the quotient algebra $B=C / \Delta$ satisfies the countable chain condition (c.c.c.) it is in fact complete. (By the c.c.c., any sup or inf reduces to a countable sub sup or subinf, and hence is well defined by the $\sigma$-completeness of B.)

We show first that $\left\|2^{\aleph_{0}} \geqslant \aleph_{2}\right\|=1$. Firstly, what do the symbols $\aleph_{0}$ and $\aleph_{2}$ mean here. Well, $\aleph_{0}$ is just the set of natural numbers, and then $\aleph_{1}$ is the first ordinal beyond $\aleph_{0}$ not equinumerous with $\aleph_{0}$, and $\aleph_{2}$ is the first ordinal beyond $\aleph_{1}$ not equinumerous with $\aleph_{1}$. Now, it is a standard result that if $\alpha$ is an ordinal, then $\| \dot{\alpha}$ is an ordinal $\|=1$, but $\kappa$ being a cardinal does not necessarily imply $\| \check{\kappa}$ is a cardinal $\|=1$. Certainly, $\| \aleph_{0}$ is the first infinite ordinal $\|=1$, so $\| \aleph_{0}$ is a cardinal $\|=1$, but for $\aleph_{1}$ this need no longer be the case. The point is, although $\aleph_{1}$ is a cardinal in $V, V^{B}$ contains more "sets" than $V$, and one of these sets may be a bijection between $\check{\omega}$ and that we may have to look further than $\aleph_{1}$ in $V^{B}$ in order to find "the $\aleph_{1}$ of $V^{B}$ ". However, in the present case there will be no problem, due to the following standard fact, whose proof we sketch.

Lemma 3.2. If B satisfies the c.c.c., then $\left\|\aleph_{0}=\aleph_{0}\right\|=1,\left\|\aleph_{1}=\aleph_{1}\right\|=1,\left\|\aleph_{2}=\aleph_{2}\right\|=$ 1 , etc.

Proof. (Sketch) Suppose $\left\|\check{\aleph}_{1}=\aleph_{1}\right\|<1$. Thus $\| \check{\omega}_{1}$ is a cardinal $\|<1$. Hence there must be an $f \in V^{\mathrm{B}}$ such that $\left\|f: \check{\omega} \stackrel{\text { onto }}{\longrightarrow} \check{\omega}_{1}\right\|=b>\mathbf{0}$. Then $b \leqslant \wedge_{\alpha<\omega_{1}} \vee_{n<\omega}\|f(\check{n})=\check{\alpha}\|$, so for each $\alpha<\omega_{1}$ we can find an $n=j(\alpha)<\omega$ such that $b \wedge\|f(\check{n})=\check{\alpha}\|>0$. Since $\omega_{1}$ is uncountable there is an uncountable set $A \subseteq \omega_{1}$ such that $\alpha \in A \rightarrow j(\alpha)=n$ for some fixed $n$. Let $b_{\alpha}=b \wedge\|f(\check{n})=\check{\alpha}\|$ for $\alpha \in A$. If $\alpha, \beta \in A, \alpha \neq \beta$, then $b_{\alpha} \wedge b_{\beta} \leqslant b \wedge \| f(\check{n})=$ $\check{\alpha}\|\wedge\| f(\check{n})=\check{\beta}\|\leqslant\| \check{\alpha}=\check{\beta} \|$. But since $\alpha \neq \beta,\|\check{\alpha}=\check{\beta}\|=0$. Hence $\left\{b_{\alpha} \mid a \in A\right\}$ is pairwise disjoint in B. This contradicts the c.c.c. for $B$.

A similar argument works for $\aleph_{2}, \aleph_{3}$, etc.

The above sketch indicates the way in which boolean truth values imitate the logical behaviour of the system, with, for instance, $\|\phi\| \leqslant\|\psi\|$ corresponding to $\|\phi \rightarrow \psi\|=1$, and $\wedge_{n<\omega}\|\phi(\check{n})\|$ being equal to $\|(\forall n \in \check{\omega}) \phi(n)\|$. The following lemma is also "standard", so again we simply sketch the proof. 
Lemma 3.3. $\left\|2^{\kappa_{0}} \geqslant \aleph_{2}\right\|=1$.

Proof. (Sketch) By 3.2 it suffices to show that there is no $f \in V^{\mathrm{B}}$ with

$$
\left\|f: \mathscr{P}(\check{\omega}) \stackrel{(1-1)}{\longrightarrow} \check{\omega}_{1}\right\|>\mathbf{0},
$$

for then $\|$ Card $(\mathscr{P}(\omega))>\aleph_{1} \|=1$, as required. (As usual, for each set $X, \mathscr{P}(X)$ is the power set of $X$.)

Suppose there is such an $f \in V^{\mathrm{B}}$.

For each $\alpha<\omega_{2}$, define $u_{\alpha} \operatorname{dom}(\check{\omega}) \rightarrow$ B by

$$
u_{\alpha}(\check{n})=\{p \in X \mid p(n, \alpha)=1\} / \Delta .
$$

Since $\operatorname{dom}\left(u_{\alpha}\right)=\operatorname{dom}(\check{\omega})$, we clearly have $\left\|u_{\alpha} \subseteq \check{\omega}\right\|=1$. For each $n \in \omega$,

$$
\left\|\check{n} \in u_{\alpha}\right\|=u_{\alpha}(\check{n})
$$

so

$$
(*)\left\|u_{\alpha}=u_{\beta}\right\|=\wedge_{n \in \omega}\left(u_{\alpha}(\check{n}) \Leftrightarrow u_{\beta}(\check{n})\right)=\{p \in X \mid(\forall n \in \omega)(p(n, \alpha)=p(n, \beta))\} / \Delta .
$$

(Here $\Leftrightarrow$ denotes the boolean operation defined by $p \Leftrightarrow q=(-p \vee q) \wedge(-q \vee p)$.) Now, for $\alpha \neq \beta,\{p \in X \mid(\forall n \in \omega)(p(n, \alpha)=p(n, \beta))\}$ can clearly contain no basic open set in $I$, and hence is nowhere dense. Thus by $\left(^{*}\right), \alpha \neq \beta$ implies $\left\|u_{\alpha}=u_{\beta}\right\|=0$.

Now, for each $\alpha<\omega_{2}$ we can pick a $\xi(\alpha)<\omega_{1}$ such that $\| f\left(u_{\alpha}\right)=\overline{\xi(\alpha) \|}=b_{\alpha}>\mathbf{0}$ and $b \wedge b_{\alpha}>0$. For a subset $A \subseteq \omega_{2}$ of cardinality $\aleph_{2}$, we must have $\xi(\alpha)=\xi$ for all $\alpha \in A$, some fixed $\xi<\omega_{1}$. Then, for $\alpha \in A, b \wedge b_{\alpha} \leqslant\left\|f\left(u_{\alpha}\right)=\check{\xi}\right\|$, so for $\alpha, \beta \in A, \alpha \neq \beta$,

$$
\begin{aligned}
b \wedge b_{\alpha} \wedge b_{\beta} & \leqslant b \wedge\left\|f\left(u_{\alpha}\right)=\check{\xi}\right\| \wedge\left\|f\left(u_{\beta}\right)=\check{\xi}\right\| \leqslant b \wedge\left\|f\left(u_{\alpha}\right)=f\left(u_{\beta}\right)\right\| \\
& \leqslant\left\|u_{\alpha}=u_{\beta}\right\|=0 .
\end{aligned}
$$

Hence $\left\{b \wedge b_{\alpha} \mid \alpha \in A\right\}$ is a pairwise disjoint subset of $\mathrm{B}$, contrary to the c.c.c. for $\mathrm{B}$.

Lemma 3.4. Assume $2^{\aleph_{0}}=\aleph_{1}$. Then $\|\Psi\|=1$.

Proof. By $2^{\aleph_{0}}=\aleph_{1}, 1.1$ implies that $\Phi$ is valid. Hence by 3.1 we can find an uncountable set $A \subseteq X$ such that $A \cap K$ is countable for every compact set $K \subseteq X$. We prove the lemma by showing that

$\|$ if $K \subseteq \omega^{\omega}$ is compact, then $\check{A} \cap K$ is countable $\|=1$.

(If $j: \omega_{1} \stackrel{(1-1)}{\longrightarrow} A$, then $\left\|\check{j}: \check{\omega}_{1} \stackrel{(1 \cdot 1)}{\longrightarrow} \check{A}\right\|=1$, so by $3.2, \| \check{A}$ is uncountable $\|=1$.) Notice that we write $\omega^{\omega}$ and not $X$ here. This is because $V^{B}$ will contain many more elements of the space $\omega^{\omega}$ than does $V$. We can, however, replace $\omega^{\omega}$ by $\check{\omega}^{\check{\omega}}$ here, and this we shall do.

Suppose that, contrary to our desires there is a $K \in V^{B}$ such that

$$
\| K \subseteq \check{\omega}^{\check{\omega}} \text { is compact and } \check{A} \cap K \text { is uncountable } \|=b>\mathbf{0} \text {. }
$$

Now, a subset of $\omega^{\omega}$ is compact iff it is a product of finite subsets of $\omega$. Let $\left\{F_{i}\right\}_{i<\omega}$ enumerate all finite subsets of $\omega$, and let 


$$
b_{n i}=\| \text { the } n \text {th component of } K \text { is } \check{F}_{i} \| \text {. }
$$

Then $i \neq j \rightarrow b_{n i} \wedge b_{n j}=0$, and

$$
\| K \text { is compact } \|=\bigwedge_{n \in \omega} \underset{i \in \omega}{V_{\text {ni }}} b_{\text {. }}
$$

For each finite map $p_{0}$ from a subset of $\omega \times \omega_{2}$ into 2, let

$$
\hat{p}_{0}=\left\{p \in I \mid p_{0} \subseteq p\right\} / \Delta .
$$

The collection of all sets $\left\{p \in I \mid p_{0} \subseteq p\right\}$ as $p_{0}$ varies is a base for the topology on $I$, so the collection of all $\hat{p}_{0}$ is a dense subset of B. Hence, for each pair $n, i$ such that $b \wedge b_{n i}>0$ we can pick a maximal pairwise disjoint collection $\mathscr{F}_{n, i}$ of elements $d \leqslant b \wedge b_{n i}$ such that each $d$ is non-zero and of the form $\hat{p}_{d}$ for some finite function $p_{d}$ from a subset of $\omega \times \omega_{2}$ into 2 . Since B satisfies the c.c.c., $\mathscr{F}_{n, i}$ is countable. Hence for each pair $n, i$ with $b \wedge b_{n i}>0$ we can find a sequence $\left\{p(n, i)_{i}\right\}_{i<\omega}$ of finite functions from subsets of $\omega \times \omega_{2}$ into 2 such that $\left\{\hat{p}(n, i)_{j} \mid j<\omega\right\}$ is a maximal disjoint subset of $\left\{d \in \mathrm{B} \mid d \leqslant b \wedge b_{n i}\right\}$. Let $\sigma=\cup_{n, i, j} \operatorname{dom}\left(p(n, i)_{j}\right)$, a countable subset of $\omega \times \omega_{2}$. Let $\left\{p_{k}\right\}_{k<\omega}$ enumerate all finite functions from subsets of $\sigma$ into 2 .

For each $a \in A$ now, let $b_{a}=\|\check{a} \in K\|$. Set $A^{\prime}=\left\{a \in A \mid b_{a} \wedge b>0\right\}$. For $a \in A-A^{\prime}$, $b_{a} \wedge b=\mathbf{0}$, so $b \leqslant-b_{a}=-\|\check{a} \in K\|=\|\check{a} \notin K\|$. Hence $b \leqslant\left\|\check{A} \cap K \subseteq \check{A}^{\prime}\right\|$. So by choice of $b, A^{\prime}$ must be uncountable.

For each $k \in \omega$, let $E_{K}=\left\{a \in A^{\prime} \mid \hat{p}_{k} \leqslant\|\check{a} \in K\|\right\}$. Suppose $a \in A^{\prime}$. Then $b_{a} \wedge b>0$, so as $b \leqslant \wedge_{n \in \omega} \vee_{i \in \omega} b_{n i}$ we can find $n, i$ with $b_{a} \wedge b \wedge b_{n i}>0$. Since $b_{a} \wedge b \wedge b_{n i} \leqslant b \wedge b_{n i}$ there is a $j \in \omega$ such that $\hat{p}(n, i)_{j} \wedge b_{a} \wedge b \wedge b_{n i}>0$. By definition of the algebra $\mathrm{B}$, it follows that we can find a $k \in \omega$ with $\hat{p}_{k} \leqslant \hat{p}(n, i)_{j} \wedge b_{a} \wedge b \wedge b_{n i}$, with $\operatorname{dom}\left(p_{k}\right) \subseteq$ $\operatorname{dom}\left(p(n, i)_{j}\right)$. Since $\hat{p}_{k} \leqslant b_{a}, \hat{p}_{k} \leqslant\|\check{a} \in K\|$, so $a \in E_{k}$. Hence $\bigcup_{k \in \omega} E_{k}=A^{\prime}$. It follows that for some $k, E_{k}$ is uncountable. Fix such a $k$ from now on.

We claim that there are integers $N_{n}, n \in \omega$, such that $E_{k} \subseteq \Pi_{n \in \omega}\left[0, N_{n}\right]$. Let $n \in \omega$ be given. Now, $\hat{p}_{k} \leqslant b \leqslant \wedge_{n \in \omega} \vee_{i \in \omega} b_{n i}$, so for some unique $i_{n} \in \omega, \hat{p}_{k} \wedge b_{n i_{n}}>0$. Let $N_{n}=\max (F)$. We show that $\left\{N_{n}\right\}_{n<\omega}$ so defined is as required.

Let $a \in E_{k}$. Fix $n \in \omega$. Since $\hat{p}_{k} \wedge b_{n i_{n}} \leqslant b_{n i_{n}}, \hat{p}_{k} \wedge b_{n i_{n}} \leqslant\left\|\check{a}_{n} \in \check{F}_{i_{n}}\right\|$. Thus $\left\|\check{a}_{n} \leqslant \check{N}_{n}\right\|>\mathbf{0}$. This can only be so if $a_{n} \leqslant N_{n}$. The claim is proved.

Let $E=\Pi_{n \in \omega}\left[0, N_{n}\right]$, a compact subset of $X$. Since $E_{k} \subseteq E \cap A, E \cap A$ is uncountable. This contradiction completes the proof of Lemma 3.4.

Hence our proof of 1.3 is also complete

\section{REFERENCES}

(1) D. H. FREMLIN, In preparation.

(2) T. JECH, Lectures in Set Theory (Springer Lecture Notes 217, 1971).

UNIVERSITY OF LANCASTER 